


\title{
Políticas para promover la ciencia, tecnología e innovación en la era digital
}

Policies to promote science, technology and innovation in The Digital Age

Fecha de recepción: 28/06/ 2019

Fecha de aprobación: 17/02/2020 


\section{Resumen}

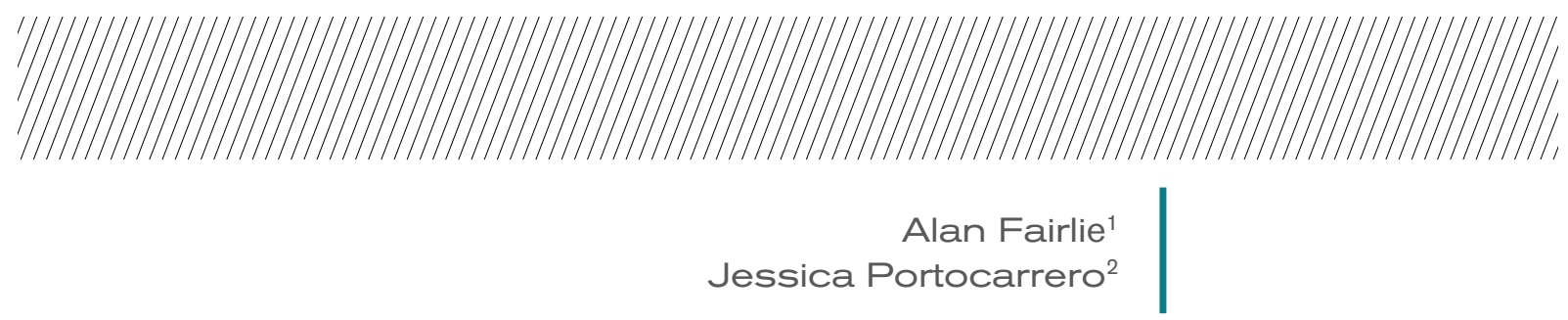

Las nuevas tecnologías se han convertido en un factor esencial para el desarrollo de los países, puesto que su uso conlleva a una serie de impactos positivos tanto a nivel económico y social. Lamentablemente, este sector se ha desarrollado a un ritmo muy lento dentro de la región andina, a consecuencia de falta de políticas públicas orientadas a desarrollar y adoptar nuevas tecnologías, por un lado; y, por el otro, existe una heterogeneidad en términos de políticas orientadas a tal fin. El documento tiene como objetivo establecer propuestas de políticas públicas orientadas a fomentar el uso de la Ciencia, Tecnología e Innovación (CTI) en los países andinos, en aras de alcanzar un desarrollo sostenible e inclusivo en la región.

Palabras clave:

Ciencia; Innovación, Lineamientos de Política; Políticas Públicas; Región Andina, Tecnología.

1 NOTA: Este artículo fue elaborado a partir de documento base del "Marco Normativo para el Fomento de Políticas Públicas de Ciencia, Tecnología e Innovación en la Región Andina", elaborado por el parla- 


\section{Abstract}

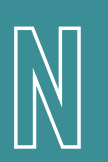

ew technologies have become an essential factor for the development of countries, since their use leads to a series of positive impacts both at an economic and social level. Unfortunately, this sector has developed at a very slow pace within the Andean region, because of a lack of public policies aimed at developing and adopting new technologies on the one hand; and on the other, there is a heterogeneity in terms of policies oriented to that end. The document aims to establish proposals for public policies aimed at promoting the use of STI in the Andean countries, in order to achieve sustainable and inclusive development in the region.

Keywords: Science; Innovation, Policy Guidelines; Public politics; Andean Region, Technology.
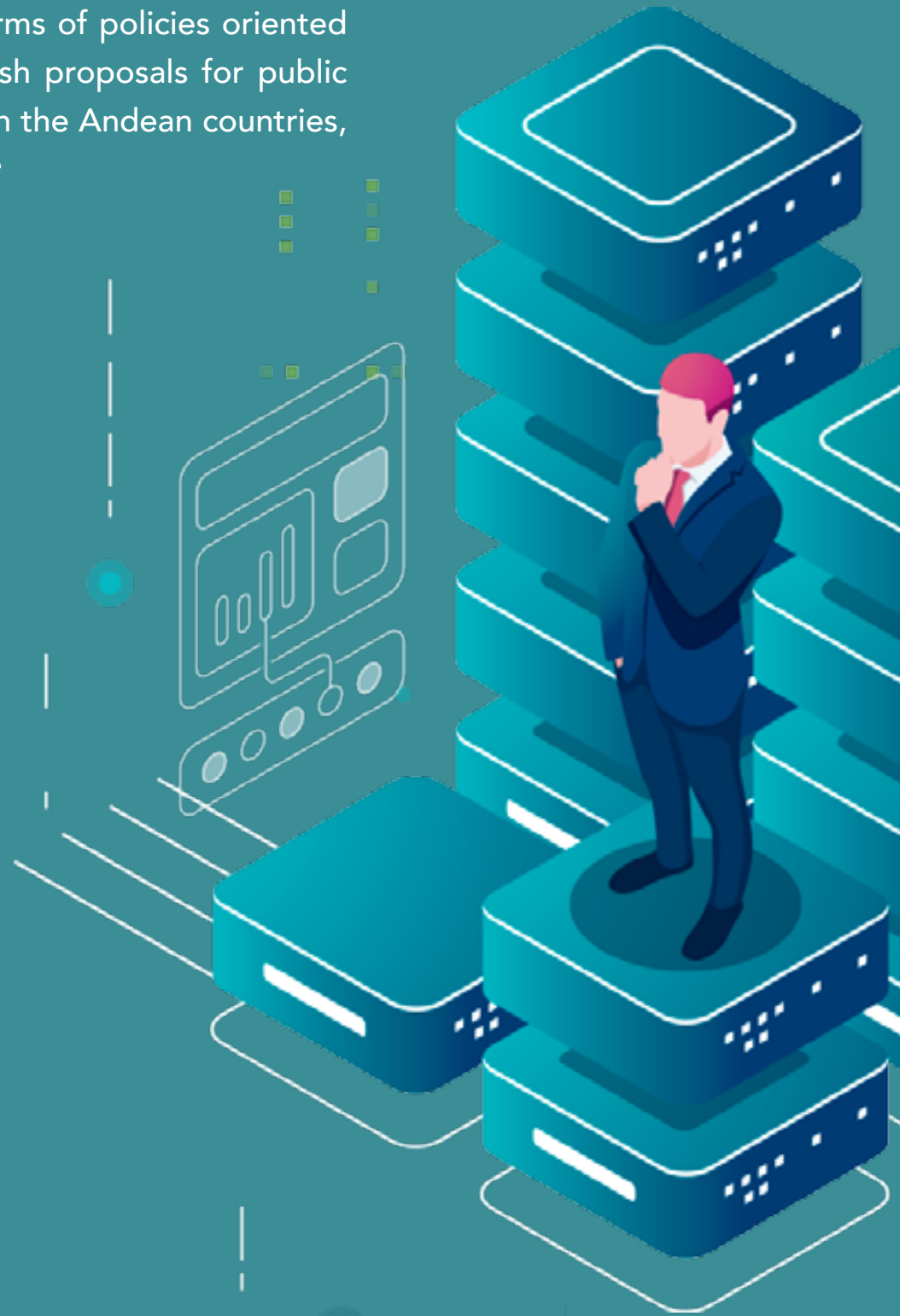


\section{Introducción}

$\mathbb{N}$

os encontramos en medio de una nueva revolución tecnológica, impulsada por la Internet móvil y la computación en la nube, la analítica de grandes datos, la Internet de las cosas, entre otros, trayendo consigo una gran repercusión en los patrones de consumo, la forma de producir y comercializar los bienes y servicios, así como en las cualificaciones y perfiles de trabajo. Sin embargo, los países en desarrollo aún no aprovechan del todo estas tecnologías para lograr transformar sus estructuras productivas y reducir las brechas en materia de competitividad y diversificación económica. Por lo cual, es indispensable que los países de la región apliquen políticas que permitan fortalecer las capacidades en materia de ciencia y tecnología. Durante el presente documento se hace una recopilación de diversos a autores sobre la importancia de la Ciencia, Tecnología e Innovación (CTI) para las economías, así como la necesidad de aplicar políticas públicas que promuevan en uso de la CTI, como medio para alcanzar un crecimiento sostenible a largo plazo.

El estudio está enfocado en los países de la región andina, los que se caracterizan por tener un modelo económico basado en la explotación de recursos naturales, presentando, además, un bajo nivel de inserción en las cadenas globales de valor, lo que no les permite lograr un crecimiento económico sostenido.

Los países andinos se encuentran rezagados en términos de CTI y presentan grandes desafíos en este campo, como los veremos en el desarrollo de este artículo; los cuales, deben ser superados a fin de propiciar la transición de los Estados hacia economías basadas en el conocimiento, con mayor valor agregado en sus procesos que permita el despegue de sectores económicos claves para el desarrollo de sus economías; por ello, se propone una serie de lineamientos de política orientados al fomento de la CTI como una herramienta que permita el logro de los Objetivos de Desarrollo Sostenible. 


\section{Desarrollo}

\section{La era digital:}

\section{principales portunidades y desafíos}

La digitalización y el uso de las nuevas tecnologías se han convertido en un factor relevante para el desarrollo económico y social de América Latina, así como de la región andina; pues, abren oportunidades para la innovación en la prestación de servicios y en modelos de negocio, dando lugar a nuevos procesos de producción, además de aumentar los niveles de productividad; generando grandes oportunidades para alcanzar el desarrollo sostenible que los países necesitan.

De acuerdo con las estimaciones del Banco de Desarrollo de América Latina (CAF), para el periodo comprendido entre el 2005 y 2014, la digitalización contribuyó con 208,7 mil millones de dólares al Producto Interno Bruto (PIB) de América Latina, lo que podría ser aún mayor debido a que los países de la región todavía no han desarrollado el gran potencial de sus entornos digitales; tal como se refleja en el Índice de Desarrollo del Ecosistema Digital, de la CAF, en donde los países latinoamericanos apenas alcanzan un puntaje de 45.47, muy por debajo del promedio de los países de la OCDE (índice: 68.94); $y$, en el caso de la región andina, este índice se encuentra incluso por debajo del promedio de América Latina.
Por otro lado, entre las principales barreras que los países de la región tienen para acceder a las oportunidades y beneficios de la economía digital, están: el déficit en cuanto a inversión en materia de infraestructura, en especial, de telecomunicaciones, en donde la inversión per cápita acumulada de cinco años, representa apenas la mitad de lo que los países de la OCDE invierten en este sector, lo cual, ha conllevado a que el " $46 \%$ de la población no tenga acceso a los servicios derivados de la conectividad, tales como telemedicina, teleducación, gobierno en línea, banca electrónica" (CAF, 2018).

En esa misma línea, diversos expertos sostienen que entre las principales limitantes para desarrollar un mercado digital en la región se encuentra "la ausencia de políticas públicas regionales, marcos regulatorios nacionales demasiado heterogéneos e infraestructuras digitales que no responden a la demanda de la economía digital" (CAF, 2017a), por lo cual, existe la necesidad de unificar los marcos regulatorios. Además, es imperiosa la necesidad de promover políticas públicas orientadas a impulsar el mayor acceso a la conectividad e interconexión en la región, que faciliten el desarrollo de las industrias digitales, la formación de capital humano altamente capacitado y una mayor inversión. 


\section{Importancia de la ciencia, tecnología}

\section{e innovación para alcanzar el desarrollo} sostenible en el largo plazo 
Lamentablemente, a lo largo de las últimas décadas, la estructura productiva en la región andina se han basado en un modelo primario-exportador con poca generación de valor agregado, en lo que CAF, CEPAL y OCDE (2019), han identificado como "trampa de la productividad, debido a que la concentración de las exportaciones en sectores primarios, extractivos y con bajos niveles de sofisticación, crean una estructura exportadora que no genera encadenamientos con el resto de la economía doméstica" ( p. 10); por lo tanto, la competitividad se ve limitada a causa de los bajos niveles de adopción de tecnología.

En esa misma línea, Estevadeordal (2017), sostiene que vivimos en un punto de inflexión y de cambio estructural, en el cual, los países de la región deben posicionarse para aprovechar las ventajas del progreso tecnológico y, a la vez, adecuar sus políticas comerciales y la formación de sus recursos humanos a un contexto más desafiante.

Por lo tanto, es crucial impulsar el uso de la Ciencia, Tecnología e Innovación (CTI) para el desarrollo de nuestra economía, especialmente, en el campo de la educación, ya que el uso de las nuevas tecnologías, tienden a facilitar el acceso universal a la educación, reducir las diferencias en el aprendizaje; además, mejorar la calidad y la pertinencia de los aprendizajes. En ese sentido, es fundamental explotar el potencial de las nuevas tecnologías, puesto que el acceso a los conocimientos se hace más sencillo para todos los niños y niñas, adolescentes y jóvenes; también, propicia el desarrollo de capacidades digitales por parte de los estudiantes, a fin de alcanzar las metas de Educación 2030 y que nadie se quede atrás, como lo establece la UNESCO². En ese sentido, la CTI y la educación se interrelacionan de forma positiva, brindado las herramientas necesarias para que los estudiantes se desarrollen en economías basadas en el conocimiento, además de impulsar el cierre de brechas de capital humano.

2 Unesco (2017) 2017 Qingdao Statement: strategies for leveraging ICT to achieve Education 2030 
Por otro lado, autores como Motta (2016), resaltan la importancia de la CTI en el fomento de las cadenas de valor, destacando que estas se expandieron, producto de la aceleración del intercambio de información debido al uso de las tecnologías de información y comunicación, así como la mejora de los sistemas de transporte y logística. Es así que el uso de nuevas tecnologías permite generar el valor agregado dentro de los procesos de producción, a más de brindar mayor oportunidad para diversificar las exportaciones y generar nuevos puestos de empleos.

En ese sentido, el uso y el aprovechamiento de la Ciencia, Tecnología e Innovación (CTI) constituyen uno de los principales pilares del crecimiento sostenible a largo plazo. Por su parte, el BID (2016) sostiene que la CTI permite alcanzar niveles constantes de productividad; sin embargo, para lograrlo los países de la región deben promover una mayor diversificación productiva y sofisticación de sus competencias y procesos, a través de mayores niveles de cooperación en temas de innovación y tecnología, tarea que aún está pendiente en los países de la región andina.

Ante este contexto, se requiere avanzar en reformas estructurales para impulsar su crecimiento potencial de las economías. En ese sentido, tal como lo señala la OCDE (2014), el fortalecimiento de la innovación es clave, así como la acumulación de competencias en las nuevas tecnologías, en aras de alcanzar la sostenibilidad económica, social y ambiental. Por lo cual, resulta fundamental que la CTI se convierta en una prioridad nacional y regional en los países andinos, partiendo por establecer y actualizar las agendas de políticas en materia de CTI. 


\section{Diagnóstico de la CTI en los países andinos}

\section{Gastos en investigación y desarrollo (I+D)}

Uno de los principales indicadores a la hora de hablar sobre CTI es el gasto en I+D, e incluso es parte de los Objetivos de Desarrollo Sostenible - ODS 9, instando a los gobiernos a promover la industrialización y la innovación sostenible, mediante el rápido incremento del gasto en I+D.

En el caso de la región andinas se ha mostrado algunos avances en materia de inversión en I+D, pero, sus niveles continúan muy por debajo al de los países desarrollados, tal como se aprecia en el grafico $\mathrm{N}^{\circ} 1$.

Gráfico N $^{\circ}$ : Gastos en investigación y desarrollo (I+D), 2016 (\% del PBI).

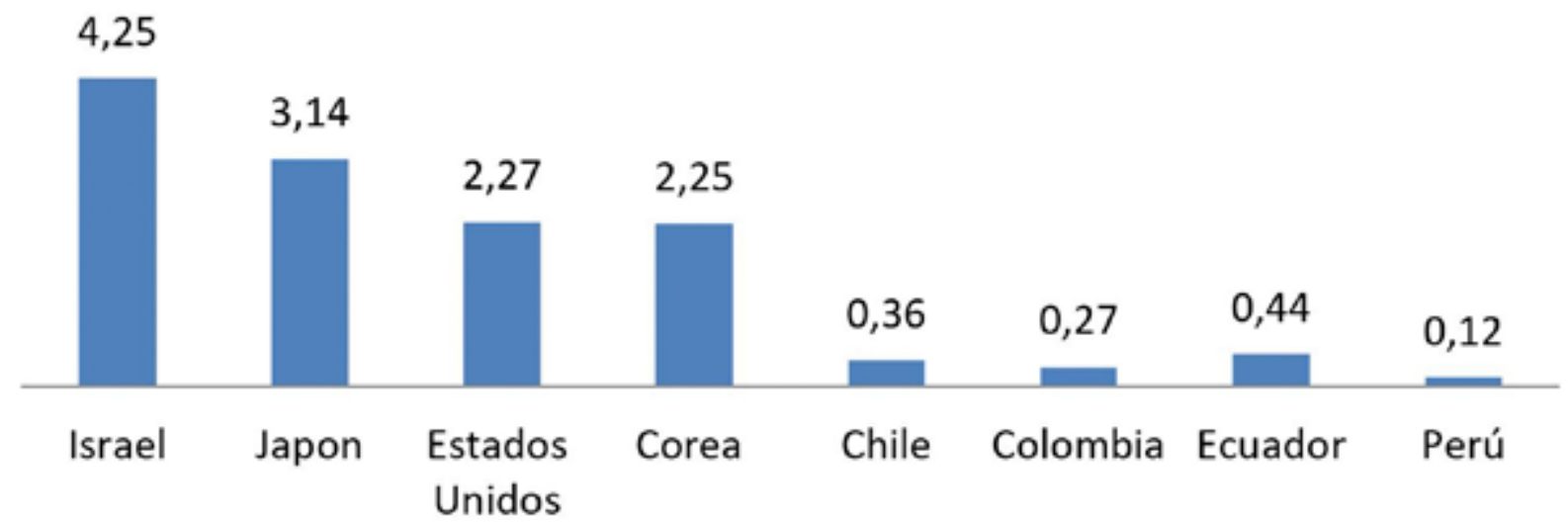


Por otro lado, según la Red de Indicadores de Ciencia y Tecnología - RICYT (2019a), en la región el gasto en I+D es financiado, principalmente, por el sector público, siendo el gobierno quien financia en promedio el $50 \%$ del total en casi todos los países; es así que el gobierno de Ecuador financia alrededor del $73 \%$ del gasto en I+D, contrario a Colombia en donde las empresas, tanto públicas como privadas, son quienes financian en mayor proporción (48\%) el gasto en I+D.

Gráfico $\mathbf{N}^{\circ}$ 2: estructura del gasto en I+D por sector de financiamiento, 2016



Fuente: RICYT (2019) / Elaboración: Propia (2019)

Por otra parte, el Gasto en I+D es ejecutado, principalmente, por el sector de educación superior. De acuerdo a la CAF (2017b), solo el $20 \%$ de las empresas latinoamericanas invierte en innovación, contrario a los países miembros de la OCDE, en donde alrededor del $60 \%$ de la inversión proviene de las empresas. Por lo tanto, el escaso involucramiento del sector empresarial con la ciencia, tecnología e innovación, es uno de los principales retos que la región debe de afrontar. 


\section{Recursos humanos dedicados a I+D}

Si se analiza la cantidad de personal dedicado a generar Investigación y Desarrollo (I+D), entre el 2011 y 2016 , se puede apreciar que se ha incrementado en un 74, pasando de 25 mil investigadores en el 2011 a más de 43 mil investigadores en 2016 (RICYT, 2019b). Sin embargo, aún persiste la brecha de género, ya que aproximadamente el 36\% de los investigadores son mujeres dentro de la región andina.

Cuadro $N^{\circ}$ 1: Países Andinos: Número de investigadores por país entre 2011-2016

\begin{tabular}{|l|r|r|c|}
\hline \multicolumn{1}{|c|}{ Investigadores por país } & 2011 & 2016 & $\%$ \\
\hline América Latina y El Caribe & 443,780 & 519,376 & $17 \%$ \\
\hline Colombia & 8,011 & 13,001 & $62 \%$ \\
\hline Chile & 9,388 & 14,200 & $51 \%$ \\
\hline Ecuador & 4,027 & $11,410 *$ & $183 \%$ \\
\hline Perú & 1,128 & $3,374^{\star *}$ & $199 \%$ \\
\hline Bolivia & 2,507 & $1,618^{*}$ & $-35 \%$ \\
\hline
\end{tabular}

Fuente: RICYT (2019) / Elaboración: Propia (2019)

* dato para 2014 / ** dato para 2015

A nivel internacional, los países de la región se encuentran en desventaja en cuanto al número de investigadores, es así que por cada mil integrantes de la Población Económicamente Activa (PEA) en los países andinos, solo hay en promedio 0.84 investigadores, mientras que el promedio de América Latina y El Caribe (ALC) es de 1.69. Por países, la cifra se distribuye de la siguiente manera: Bolivia (0.3), Colombia (0.5), Chile
(1.6), Ecuador (1.6), Perú (0.2). Cabe señalar que en este indicador estamos aún muy lejos de los países de la OCDE, con un promedio de 12.7 (RICYT, 2019c). Asimismo, en el 2016, el $35.1 \%$ de los investigadores andinos tenía grado de doctor, cifra que se ubica por debajo de otros países de la región, como es el caso de Uruguay en que este porcentaje es $60 \%$, mientras que, en la OCDE, este porcentaje se ubica en $42.8 \%$. 


\section{Publicaciones científicas}

Durante el último quinquenio el número de artículos publicados por los países andinos en revistas científicas, en la base SCOPUS, ha pasado de 17,768 en el 2012 a 28,303 en el 2016, lo que representa un crecimiento de $59 \%$ y una producción científica acumulada de 112,396 publicaciones.

Si bien Chile es el país que ha registrado mayor número de publicaciones para el periodo de referencia, con un total de 53,777 publicaciones, se destaca el crecimiento de Ecuador, que logró aumentar en $270 \%$ la cantidad de publicaciones en esta base de datos. No obstante, para el mismo periodo, los países de la región solo representaron el $18 \%$ del total de publicaciones en América Latina y El Caribe (RICYT, 2019d).

Cuadro № 2: Número de producciones afiliadas por país, 2012 -2016

\begin{tabular}{|c|c|c|c|c|c|c|}
\hline Países & 2012 & 2013 & 2014 & 2015 & 2016 & 2012-2016 \\
\hline Estados Unidos & 646,221 & 652,300 & 652,345 & 655,507 & 641,969 & $3,248,342$ \\
\hline $\begin{array}{l}\text { América Latina y El } \\
\text { Caribe }\end{array}$ & 112,090 & 118,043 & 126,626 & 128,619 & 136,423 & 621,801 \\
\hline Países andinos & 17,768 & 19,346 & 22,396 & 24,583 & 28,303 & 112,396 \\
\hline Bolivia & 252 & 283 & 310 & 322 & 330 & 1,497 \\
\hline Chile & 8,907 & 9,321 & 11,000 & 11,573 & 12,976 & 53,777 \\
\hline Colombia & 6,585 & 7,445 & 8,318 & 8,999 & 10,239 & 41,586 \\
\hline Ecuador & 648 & 762 & 1,047 & 1,657 & 2,395 & 6,509 \\
\hline Perú & 1,376 & 1,535 & 1,721 & 2,032 & 2,363 & 9,027 \\
\hline
\end{tabular}

Fuente: RICYT (2019) / Elaboración: Propia (2019) 


\section{Patentes}

El número de patentes solicitadas por residentes y no residentes, en las oficinas nacionales de los países andinos entre 2007 y 2016, se redujo en $21 \% 3$, provocado por la caída en Ecuador (-40\%), Chile (-24\%) y Perú (-14\%). Solo Colombia incrementó en $8 \%$ en número de las patentes solicitadas. De otro lado en el 2016, de 6,273 solicitadas solo fueron otorgadas 3,394, es decir, el 54\% del total (RICYT, 2019e).

Cuadro N 3: Países Andinos: Número de patentes solicitadas de acuerdo a tipo de residencia, 2016

\begin{tabular}{|c|c|c|c|c|}
\hline Solicitudes de Patentes & Chile & Colombia & Ecuador & Perú \\
\hline De residentes & $13 \%$ & $25 \%$ & $10 \%$ & $6 \%$ \\
\hline de no residentes & $87 \%$ & $75 \%$ & $90 \%$ & $94 \%$ \\
\hline
\end{tabular}

Fuente: RICYT (2019) / Elaboración: Propia (2019)

En la región, las patentes solicitadas por los residentes representan un porcentaje muy pequeño, siendo que para el periodo 2007-2016 este número se ubica entre el 7\% y 15\% del total de patentes solicitadas ${ }^{4}$; contrario a lo que sucede en países desarrollados, como los Estados Unidos en donde esta proporción es más del 50\% (RICYT, 2019f).

3 Nota: El cálculo toma los datos de RICYT (2019) y se considera a los siguientes países: Chile, Colombia, Ecuador y Perú, puesto que, no hay datos disponibles para Bolivia.

4 El porcentaje de patentes solicitadas por los residentes de cada uno de los países andinos fue calculado en base a los datos de RICYT (2019 e), véasehttp://app.ricyt.org/ui/v3/comparative.html?indicator=CPATSOL\&start_year=2008\&end_year $=2017$ 


\section{Importancia de las políticas de Ciencia, Tecnología e Innovación para el Desarrollo Sostenible}

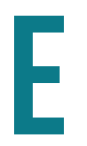

En el contexto en el que nos encontramos, una adecuada política de CTI en los países andinos, permitirá contar con personas cada vez más capacitadas, procesos de producción más sofisticados, pues, la inserción su inserción en el mercado global depende en gran parte de las capacidades científicas y tecnológicas y su habilidad para adoptar nuevos métodos de producción.

Si bien los países de la región andina cuentan con políticas, planes y programas de $\mathrm{CTI}$, alineados a las prioridades nacionales, algunos de estos se encuentran más avanzados que otros, tal como pudimos ver en el capítulo anterior, donde se pudo observar que nos encontramos rezagados en comparación con las economías desarrolladas e, incluso, con algunos países de América Latina, como consecuencia de la débil relación entre ciencia, tecnología e innovación y las políticas. Asimismo, no hay avances significativos en la evaluación de políticas de CTI y su impacto, además de que los sistemas nacionales de innovación están poco integrados, tal como los señala CEPAL (2010).
Las políticas de CTI en la región, históricamente han estado enfocadas en políticas científicas y en la oferta de becas para la formación e investigación de la comunidad científica; primero, en el extranjero y, luego, a nivel nacional, aunque no se ha visto a la ciencia como una fuente de contribución a la economía, lo que quiere decir que existe una fragmentación entre las políticas de ciencia y tecnología.

En ese sentido, las políticas públicas deben estar orientadas a promover la trasformación productiva y del desarrollo sostenible e impulsar la cooperación entre los agentes y la institucionalidad, así como instrumentos basados tanto en la oferta como en la demanda de CTI. En la siguiente sección se hace un balance de las políticas públicas en materia de CTI que los países andinos. Dadas sus características, deben de impulsar en aras de alcanzar el desarrollo sostenible y consolidar los ecosistemas de innovación y producción en la región. 


\section{Propuestas de lineamientos de políticas de CTI}

Para lograr que las CTI contribuyan en el crecimiento a largo plazo de las economías, elevando los niveles de competitividad de los países y, al mismo tiempo, estén al servicio de la sociedad y favorezcan en la equidad social, se requieren cambios estructurales donde las políticas estén articuladas entre ellas.

\section{(A) Impulsar la generación de fuentes de recursos financieros}

destinados a actividades de CTI. Es necesario que los países de la región andina destinen mayores recursos en materia de I+D como porcentaje del PBI. Asimismo, se debe de promover mayores niveles de inversión en CTI, a través de una política de atracción de inversión extranjera hacia actividades intensivas en tecnología; además, establecer beneficios tributarios a fin de promover la inversión privada en investigación y desarrollo.

B Formación de recursos humanos altamente capacitado. Se debe de partir por el fortalecimiento del sistema educativo dentro de los países andinos, con el objetivo de promover el desarrollo de capacidades y habilidades de la población estudiantil en el ámbito de la CTI. Asimismo, es necesario impulsar la movilidad académica de investigadores a nivel de la región y fuera de ella, con el objetivo de promover el intercambio de buenas prácticas en el diseño de políticas de CTI. Además, resulta indispensable contar con becas doctorales y promover el libre acceso a bibliografía especializada. Asimismo, es necesario acortar las brechas de género a través de una mayor participación de las mujeres en actividades más sofisticadas y con base tecnológica. Finalmente, establecer una estrategia de repatriación de capital humano, brindándoles las mejores condiciones, trabajos adecuadamente remunerados. 
Establecer, mecanismos, programas y servicios de apoyo para el fomento de la CTI. Resulta indispensable propiciar una mayor articulación entre los actores vinculados a la CTI (sector público, privado, academia y sociedad civil), identificando su demanda en materia de ciencia y tecnología e impulsando la adopción de tecnologías, así como la trasferencia de conocimiento de acuerdo a cada una de sus necesidades; por lo tanto, resulta necesario el establecimiento de programas sectoriales en materia de CTI. Por otro lado, dado el escaso involucramiento entre la academia y el sector privado, es necesario establecer mecanismos de interacción a fin de promover una mayor investigación y absorción de nuevas tecnologías dentro de las empresas; además, establecer alianzas estratégicas con el establecimiento de laboratorios y centros de investigación.

D Proveer una adecuada infraestructura física e institucional. Es necesario que los países andinos establezcan un plan de mantenimiento y renovación de la infraestructura y equipos robustos en materia de CTI. Asimismo, se debe promover la interconexión en red de investigadores y académicos, con sus pares a nivel de la región e internacionalmente, a través de la creación de una plataforma y Red de Investigadores Andinos, así como el establecimiento de laboratorios y centros de investigación multidisciplinarios en la región andina.

E) Eliminar las barreras legales e institucionales existentes para promover mayores niveles de investigación científica. La región andina presenta bajos niveles de registro de patentes, en especial, las nacionales. Para revertir esta situación es necesario establecer una normativa que facilite el patentamiento y la transferencia tecnológica, además de simplificar los procedimientos de registro; asimismo, se debe de fomentar el respeto a la propiedad intelectual. Por otro lado, es importante establecer visados y regímenes laborales especiales para los investigadores extranjeros. 
F Establecer la carrera del investigador científico. Para evitar la salida de capital humano hacia el exterior, resulta indispensable promover la carrera del investigador, a través del establecimiento de concursos y convocatorias nacionales, además de instaurar categorías o niveles de incorporación a la carrera del investigador que estén en función a: grado académico, años de experiencia, número de artículos científicos publicados en revisas indexadas y el impacto de sus publicaciones.

(G) Promover la investigación e innovación en las empresas como medio para aumentar los niveles de competitividad y productividad. En la región son pocas las empresas que invierten en I+D, por lo cual, se deben establecer lineamientos de políticas orientados a fomentar el desarrollo y la transferencia de innovaciones tecnológicas dentro de las empresas, en especial, en las PYMES, a fin de establecer procesos productivos sofisticados y promover la internacionalización de las PYMES. En esa misma línea, es necesario fomentar la creación y el financiamiento de emprendimientos con base tecnológica y establecer programas de apoyo, así como políticas orientadas a la formación de cadenas productivas de valor, la creación de clústeres productivos y el desarrollo de parques tecnológicos. Por otro lado, se debe de promover el uso de compras públicas para estimular la demanda de productos innovadores e inducir al sector productivo y empresarial a invertir en investigación y desarrollo.

H Promover y facilitar la inclusión social mediante el uso de la ciencia, tecnología e innovación. Impulsar la creación de programas orientados atender a los sectores más desprotegidos mediante el uso de la ciencia, tecnología e innovación para la producción de bienes y servicios que estén alineados a las necesidades de dicho sector. Además, se debe facilitar el acceso al uso de las tecnologías a la población de los sectores más vulnerables, zonas marginales y áreas rurales, con el fin de disminuir las brechas de acceso a la información y el conocimiento. 
(1) Fomentar la investigación en áreas estratégicas. Identificar sectores estratégicos en los países andinos, en donde a través del uso y adopción de mecánicos de CTI se alcance su desarrollo potencial, contribuyendo así al crecimiento económico y el desarrollo social de la región. En ese sentido, es necesario identificar las tecnologías que son necesarias y prioritarias para las áreas estratégicas de nuestro país, lo que permitirá generar y establecer ventajas competitivas.

D Promoción y difusión de la CTI en la sociedad en su conjunto. A fin de que la sociedad pueda adaptar e incorporar estos nuevos conocimientos en sus actividades, es necesario dar a conocer el impacto del uso de la CTI en el desarrollo económico y social de los países; en ese sentido, es necesario fomentar su popularización.

( I) Institucionalidad de las CTI. En necesario que los estados cuenten con un organismo con rango ministerial que se encargue del diseño, implementación y monitoreo de políticas de CTI, además de ser el responsable de la articulación con otros sectores del Estado responsables de aspectos vinculados, tales como: educación, comercio, política industrial y propiedad intelectual, con el fin de incrementar la capacidad de respuesta a problemas productivos y sociales. 


\section{Conclusiones}

0

omo se ha visto a lo largo del presente documento, el contexto de la CTI en los países de la región andina es poco alentador: aún persisten bajos niveles de gasto en I+D, la falta de una adecuada infraestructura, así como capital humano altamente capacitado que pueda hacer frente a los cambios producto de la Cuarta Revolución Industrial.

Por otro lado, en los últimos años, los países de la región han venido incorporando débilmente la relación entre ciencia, tecnología e innovación y la interacción entre los agentes en sus políticas, sin lograr avances significativos en cuanto a la evaluación de las políticas de CTI; a esto se suma que los sistemas nacionales de innovación se encuentran poco integrados. Ante este contexto, los países de la región deben aplicar políticas que permitan el fomento y la construcción de capacidades en ciencia y tecnología coherentes con las demandas de los nuevos mercados emergentes.

En ese sentido, es necesario fortalecer la integración y la cooperación en materia de ciencia, tecnología e innovación de los países andinos, a través de políticas públicas que faciliten la transferencia del conocimiento, formación y capacitación de talento humano, la adopción de procesos productivos más sofisticados con la finalidad de buscar mayores niveles de desarrollo económico y generar productos con mayor valor agregado que permitan transitar de un modelo primario-exportador, hacia uno basado en el conocimiento y la trasferencia tecnología. 
Urge impulsar mayores niveles de inversión en CTI en los países andinos, que permitan implementar estrategias nacionales y regionales para su fomento, así como una masa crítica de científicos e infraestructura física e institucional en los países de la región. También es necesario, fomentar la innovación empresarial a través de la vinculación entre la empresa, las universidades y el Estado, en aras de crear producción tecnológica que permita a las empresas mejorar su productividad. Asimismo, se debe promover el enfoque de innovación social o inclusiva, que permita abordar las necesidades de la población, especialmente, de los grupos más vulnerables, con el fin de disminuir los niveles de pobreza, por lo cual, se debe de entender a la CTI como un elemento interdisciplinario. 


\section{BIBLIOGRAFÍA}

BID (2016). The New Imperative of Innovation Policy Perspectives for Latin America and the Caribbean. Washington, D.C.

CAF, CEPAL y OCDE (2019) Perspectivas económicas de América Latina 2019: Desarrollo en Transición

CEPAL (2010). Ciencia y tecnología en el Arco del Pacífico Latinoamericano: espacios para innovar y competir. Santiago de Chile.

CEPAL (2012), Structural Change for Equality: An Integrated Approach to Development. Comisión Económica para América Latina, Naciones Unidas, Santiago de Chile.

CEPAL (2015) La Unión Europea y América Latina y El Caribe ante la nueva coyuntura económica y social. Comisión Económica para América Latina, Naciones Unidas. Santiago de Chile.

CEPAL, CAF, OCDE (2019). Perspectivas económicas de América Latina 2019: Desarrollo en Transición.

Estevadeordal, A (2017). Estar Preparados. Robot lución: el futuro del trabajo en la integración 4.0 en América, BID- INTAL, N² (ISSN: 1995-9524). Pg. 10-11.

Motta, Marialisa (2016) Inserción en las Cadenas de Valor Internacional. Presentación en la IV Edición del Foro Industrial 2016. Sociedad Nacional de Industrias SNI, Lima, Perú.

Unesco (2017) 2017 Qingdao Statement: strategies for leveraging ICT to achieve Education 2030

\section{RECURSOS ELECTRÓNICOS:}

CAF (2019) Observatorio del Ecosistema Digital de América Latina y El Caribe. Recuperado 13 de junio del 2019, del sitio web: https://www.caf.com/app_ tic/\#es/home

CAF (2018) Cómo conectar digitalmente a la otra mitad de América Latina. Recuperado 05 de junio del 2019, del sitio web: https://www.caf.com/es/ 
actualidad/noticias/2018/04/vamos-todos-a-conectar-digitalmente-a-la-otramitad-de-america-latina/

CAF (2017a) Los beneficios sociales y económicos de un mercado digital latinoamericano. Recuperado 05 de junio del 2019, del sitio web:

CAF (2017b) Generación de capacidades de gestión de la innovación: Desarrollo de la estrategia de I+D+I para Colombia. Recuperado 05 de junio del 2019, del sitio web: https://www.caf.com/es/actualidad/noticias/2018/12/los-beneficiossociales-y-economicos-de-un-mercado-digital-latinoamericano/

Instituto de estadísticas de la Unesco (2019). Gross domestic expenditure on R\&D as a percentage of GDP. Recuperado 05 de junio del 2019, del sitio web http://data.uis.unesco.org/Index.aspx?DataSetCode=SCN_DS\&lang=en\#

RICYT (2019 a). Gasto en I+D por sector de financiamiento. Recuperado 13 de junio del 2019, del sitio web: http://app.ricyt.org/ui/v3/comparative.html?indicator $=$ GASIDSFPER\&start_year $=2008 \&$ end_year $=2017$

RICYT (2019b). Investigadores por sector de empleo. Recuperado 13 de junio del 2019, del sitio web: http://app.ricyt.org/ui/v3/comparative.html?indicator $=$ INVESTPFSEPER \&start_year=2008\&end_year=2017

RICYT (2019c), Investigadores cada 1000 de la PEA. Recuperado 13 de junio del 2019, del sitio web: http://app.ricyt.org/ui/v3/comparative.html?indicator= CINVPEA\&start_year=2008\&end_year=2017

RICYT (2019d), Publicaciones en SCOPUS. Recuperado 13 de junio del 2019, del sitio web http://app.ricyt.org/ui/v3/comparative.html?indicator=CSCOPUS\&start_ year $=2008 \&$ end_year $=2017$

RICYT (2019e), Solicitudes de Patentes. Recuperado 13 de junio del 2019, del sitio web: http://app.ricyt.org/ui/v3/comparative.html?indicator=CPATSOL\&start_ year $=2008 \&$ end_year $=2017$

RICYT (2019f), Patentes otorgadas. Recuperado 13 de junio del 2019, del sitio web: http://app.ricyt.org/ui/v3/comparative.html?indicator=CPATOTOR\&start_ year=2008\&end_year $=2017$ 